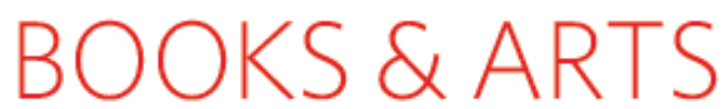

\title{
A healthy dose of genetics
}

\section{How much has genetics contributed to advances in medicine?}

\author{
Moments of Truth in Genetic Medicine \\ by Susan Lindee \\ Johns Hopkins University Press: 2005. \\ 288 pp. $\$ 40$
}

\section{Uta Francke}

The sequencing of the human genome is widely touted as a watershed event in biomedical science, and has brought an expectation that genetic information will revolutionize the practice of medicine. A reality check is in order, however. An examination from a historical viewpoint of how technical progress in medical genetics has led to clinical advances may modify our views of what 'genomic medicine' is likely to deliver.

In Moments of Truth in Genetic Medicine, Susan Lindee, a historian at the University of Pennsylvania, Philadelphia, focuses on 'case studies' in the history of human and medical genetics, emphasizing the family, social and political context of genetic disease. She examines the contributions of lay individuals and organizations to researchers' successes, and explores the connections between academic research, biotechnology, and financial and political motivations. She initially focuses on landmark developments between 1955 and 1975 , but brings the story to the present day. She critically assesses the role of gene discoveries in finding cures and the potential for gene therapy. And she declares that the selective abortion of affected fetuses following prenatal genetic diagnosis is the "primary intervention of genomic medicine" to date. The more technically and financially demanding pre-implantation genetic diagnosis and embryo selection are not discussed.

The five case studies are well chosen to illustrate different aspects. 'Two Peas in a Pod' focuses on the use of twin research to determine the genetic component of human behaviour, exemplified by studies of alcoholism. 'Baby's Blood' explains in detail how the Guthrie blood-spot diagnostic test for phenylketonuria led to the establishment of screening programmes for newborns, with the aim of identifying affected homozygotes and instituting dietary treatment to prevent the development of mental retardation. Curiously, the implications of screening results for recurrence risk and carrier status of other family members are not mentioned. One wonders whether these genetic implications were even

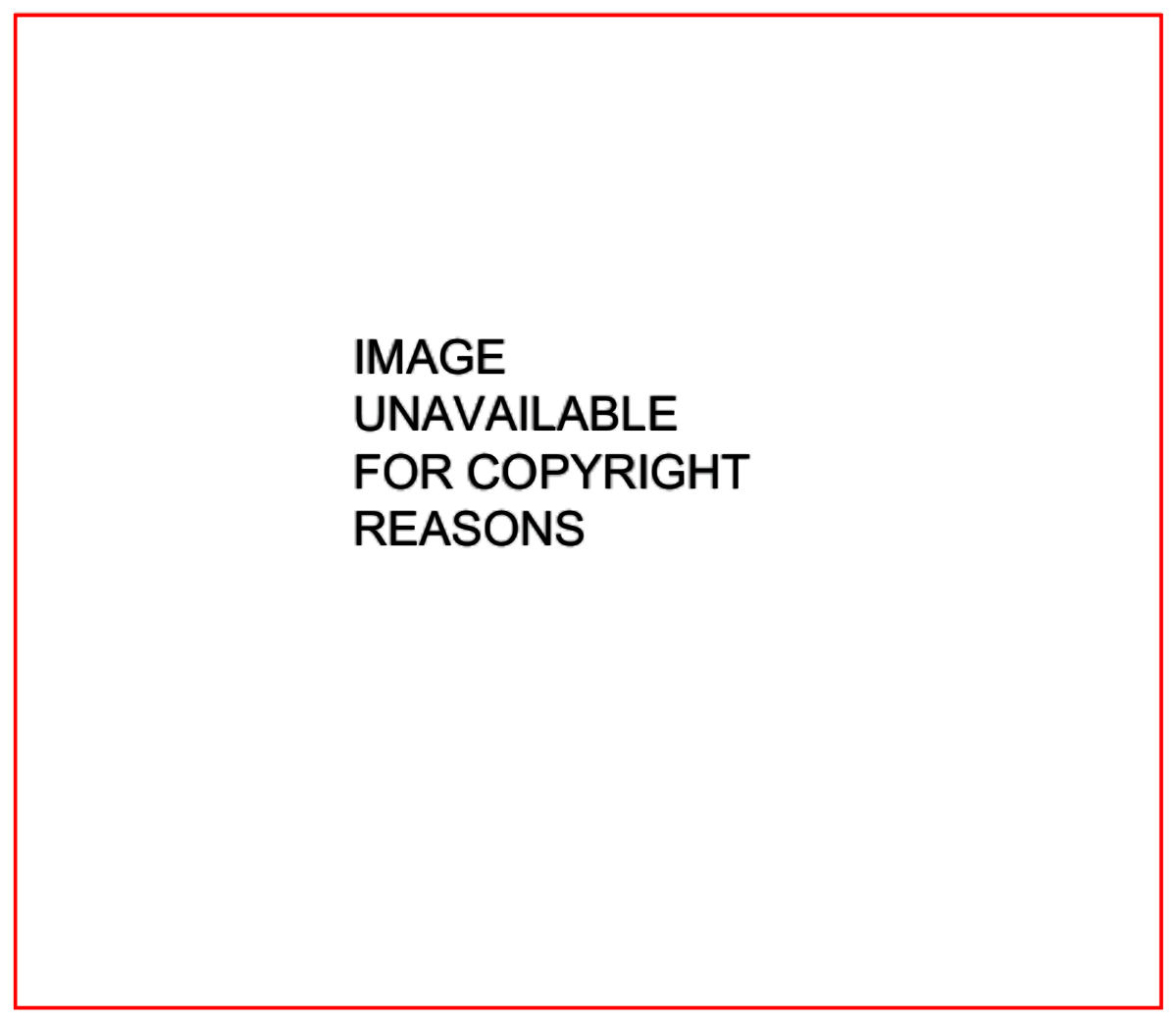

A seven-day-oldbaby is given the Guthrie test for detecting phenylketonuria.

made clear in the consent forms in the 1960s.

'Squashed Spiders' conveys the excitement about the discovery that having the wrong number of chromosomes can cause dinical syndromes. Karyotyping was the first widely used genetic diagnostic test for developmental disorders, and drew paediatricians, in particular, into the emerging field of medical genetics. Once the correct human chromosome number was established, an international effort to standardize the human karyotype was undertaken, even before most chromosomes could be unequivocally identified. Unfortunately, Lindee fails to explain correctly which chromosomes were individually identifiable and which were not. The international committee set up to standardize human cytogenetics subsequently developed numbering systems for chromosome bands revealed by different techniques.

'Provenance and the Pedigree' describes Victor McKusick's work with an inbred population in Pennsylvania: the Amish. The story of a rare, inherited form of dwarfism with extra fingers exemplifies the social, emotional and technical aspects of collecting pedigree information. Lindee beautifully illustrates the personal relationship that develops between the researcher and the people who agree to divulge information about their family and medical history that is essential for the research to succeed. With the pedigree as the "bedrock tool of human molecular genetics", the people are not only research subjects but collaborators too. From the perspective of today's human geneticist, encumbered by increasing regulatory requirements and restrictions, it is amazing to see how easygoing and casual the interactions were when McKusick and his colleagues made their field trips into Amish country.

An ongoing contribution from McKusick is the collection of information on mendelian traits. First published in 1966 as Mendelian Inheritance in Man, this database is continually updated online, where it is known as Online Mendelian Inheritance in Man (OMIM). Lindee says the motivation for the creation of this catalogue was the collection of literature reports for the naming and classifying of 
clinical syndromes to guide genetic counselling. McKusick's major purpose, however, was to create a list of traits that could subsequently be mapped. In this book, the term 'mapping' has several meanings and is often used synonymously with 'sequencing.' The correct definition of mapping is either to place genes into a linear order (genetic mapping) or to assign them to chromosomal regions (physical mapping). The contribution of OMIM to gene mapping and the subsequent completion of the human genome sequence is immense.

In 'Jewish Genes', Lindee tells the story of familial dysautonomia, a rare recessive disorder characterized by lack of tears, autonomic dysfunction and emotional lability. Starting with the delineation of the phenotype and the development of a diagnostic skin test, she then discusses the mapping of the location of the disease gene and prenatal molecular testing by linkage analysis in affected families, and finally the identification of the mutant gene and its function. She allows the reader to enter the body of an affected individual and imagine living with the disease. It has highly variable clinical manifestations, induding episodes of fever and high blood pressure, impaired taste, vomiting, insensitivity to pain, undue reaction to anxiety and lack of motor coordination. The story of familial dysautonomia also focuses on the interactions between foundations that distribute research money, collected from affected families and their friends, and researchers who accept the funds and commit to work on the disease. The spectrum of interactions ranges from mutual support and respect to competitiveness and secretive behaviour.

These fascinating, well-written stories portray what it is like to work in human or medical genetics, both in the clinic and as a researcher. Nowadays, medical genetics is an established medical speciality, unlike 'genomic medicine, which is a poorly defined futuristic term. Students and researchers in genetics and other fields of biomedicine are likely to gain new perspectives on their own work by reading this book. And because everybody is a healthcare consumer, general readers may well identify with certain actors in these stories. Uta Francke is in the Department of Genetics, Stanford University School of Medicine, Stanford, California 94305-5323, USA.

\section{In the minds of animals}

Animal Passions and Beastly Virtues:

Reflections on Redecorating Nature

by Marc Bekoff

Temple University Press: 2005.320 pp.

$\$ 70.50$ (hbk), $\$ 26.95$ (pbk)

\section{Scott Creel}

In T. H. White's The Once and Future King (G. P. Putnam, 1958), Merlin educates a young King Arthur by transmogrifying him into other creatures. Sometimes Arthur is an ant, and considers everything in the world only as 'done' or 'not done'. Sometimes he is a falcon, overcome in mid-sentence by a desire to kill his conversational partner. For many of us, the game of wondering 'What is it like to be a wildebeest?' never loses its charm. In Animal Passions and Beastly Virtues, Marc Bekoff explores this question in the context of cognitive ethology.

The book is really a collection of papers that Bekoff published between 1977 and 2004 on five main topics. The sections on social behavior in canids and on the functions of play fit neatly into conventional behavioural ecology. The quality of these original papers varies, from an excellent and influential paper on the social ecology of coyotes to a paper on scent-marking with a single subject, Bekoff's dog Jethro.

In three sections on animal minds, interactions between humans and animals, and the ethics of studying animals, Bekoff skates boldly on to thin ice at the edges of science and philosophy. In the section on emotions, cognition and animal selves, Bekoff argues that many species have minds, emotions and a sense of self that bear recognizable similarities to those of humans. As well as providing case studies, Bekoff argues that invoking the existence of emotions or intentions often provides the most parsimonious explanation of behaviour. $\mathrm{He}$ also notes that the selection pressures that produced cognition and emotion in humans are shared by social carnivores, primates and birds, and he applies the principle of evolutionary continuity to suggest that similar outcomes may have arisen in the minds of other species. In addition to the more conventional argument that homologues of emotion are likely in non-human apes, he argues that convergent evolution may have produced emotions in species more distantly related to humans. This is thought-provolking material for anyone who wonders what the world is like for other species.

The book focuses mainly on Bekoff's work with dogs. Readers seeking broader surveys might also be interested in Animal Social Complexity edited by Frans de Waal and Peter Tyack (Harvard University Press, 2003), or Why Men Won't Ask For Directions by Richard C. Francis (Princeton University Press, 2003).

Bekoff's essays on the interactions between humans and other species raise important questions for those who study animals or who simply care about their conservation and wellbeing. The section on anthropogenic effects focuses on Bekoff's work with prairie dogs and domestic dogs. These papers are informative but for a broader view of the impact of humans on animals, the interested reader might also look at Wildlife and Recreation by R. L. Knight and K. J. Gutzwilller (Island Press, 1995).

The ethics of our interactions with other species provides the basis for some challenging discussions. Bekoff often concedes that these issues cannot be resolved, but he makes a compelling case that the questions are worth our attention. He argues that we should apply the precautionary principle to our interactions 